\title{
A rapid screening test for reduced fibrinolytic activity of plasma: streptokinase activated lysis time
}

\author{
E. GIDRON, R. MARGALIT, AND Y. SHALITIN \\ From the Clinical Research Laboratory, Department of Medicine B, Rothschild University Hospital and \\ Department of Biology, Technion-Israel Institute of Technology, Haifa, Israel
}

SUMmARY A simple screening method for determining the fibrinolytic activity of plasma is described. A streptokinase activated system is used, which measures the result of the interplay of all components of the fibrinolytic system with the exception of activators, which are added in excess. Mean lysis time and standard deviation with this method is $6.9 \pm 1.4 \mathrm{~min}$. The sensitivity of the method in detecting minor delays in clot lysis time is demonstrated.

The methods available for the determination of reduced fibrinolytic activity of plasma are few and are not easily applied to individual cases owing to wide limits of normal. The dilute blood clot lysis time (Fearnley et al., 1957) has an arbitrary normal limit of 7 hours, but many normal clots lyse after 3-4 hours; for them 7 hours is an abnormal value. Similarly, the euglobulin lysis time (von Kaulla and Schultz, 1958) has a normal range of 2.5 to 4 hours, representing the lysis time of a clot with most inhibitors removed. In the fibrin plate method (Astrup and Müllertz, 1952) the area of lysis is measured, all the inherent inaccuracies of such measurements being increased by the small size of the area when lysis is delayed. Hickman's (1971) method using labelled fibrinogen is accurate but rather cumbersome.

The purpose of this paper is to describe a simple method for the determination of the fibrinolytic activity of plasma in an activated system. This method reflects the influence of all the components of the complex fibrinolytic system with the exception of activators, which are added in excess.

The method is based partly on the observations of Konttinen (1965), who investigated the influence of various concentrations of streptokinase (SK) on plasma clot lysis time, and on the urokinase (UK) sensitivity test of McNicol et al. (1963).

\section{Materials}

1 Streptokinase (Varidase $^{R}$, Lederle): The stock solution containing $10000 \mathrm{U}$ of SK per $\mathrm{ml}$ of phosphate buffer was kept at $-20^{\circ} \mathrm{C}$ and was stable for two weeks.

Received for publication 8 July 1977
2 Thrombin (Sigma): The stock solution containing $50 \mathrm{U} / \mathrm{ml}$ of phosphate buffer was kept at $-20^{\circ} \mathrm{C}$ in aliquots of $2 \mathrm{ml}$ and was stable for two weeks.

3 Urokinase ${ }^{1}$ : Aliquots of $2 \mathrm{ml}$ containing 480 CTA units per $\mathrm{ml}$ were stored in the dark at $0^{\circ} \mathrm{C}$. 4 Human Fibrinogen (Kabi): coagulability of $95 \%$.

5 Plasminogen (Profibrinolysin, Sigma): Aliquots of $1 \mathrm{ml}$ containing $5 \mathrm{U}$ of plasminogen were stored at $0^{\circ} \mathrm{C}$.

6 Phosphate buffer: $\mathrm{pH} 7 \cdot 4,0 \cdot 15 \mathrm{M}$.

\section{Methods}

1 Blood sampling: $4.5 \mathrm{ml}$ of blood was drawn from fasting subjects between 0800 and 0900 hours and placed in tubes containing $0.5 \mathrm{ml}$ of sodium citrate $(3 \cdot 8 \%)$.

2 Fibrinogen was determined by the method of Godal (1961). The normal range with this method is $2 \cdot 0-4 \cdot 0 \mathrm{~g} / 1$.

3 Plasma streptokinase lysis time: $0.1 \mathrm{ml}$ streptokinase in concentrations of $100,150,200,500$, 1000 , and $2500 \mathrm{U} / \mathrm{ml}$ of plasma was added to test tubes containing $0.1 \mathrm{ml}$ of plasma and $0.8 \mathrm{ml}$ of phosphate buffer, and the mixture was clotted by an addition of $5 \mathrm{U}$ of the stock solution of thrombin. The lysis time was defined as the time elapsing from the moment of adding thrombin until complete lysis of the clot.

4 Dilute blood clot lysis time was performed by the method of Fearnley et al. (1957). The end point was determined visually or photographically;

${ }^{1}$ Kindly supplied by Dr Alan Johnson 
the normal range in 35 controls was in our laboratory 3-5 hours.

5 Streptokinase activated lysis time (SALT): The blood samples were centrifuged at room temperature for $30 \mathrm{~min}$ at $3000 \mathrm{rpm}$, and the plasma was separated. $0.15 \mathrm{ml}$ of a $100 \mathrm{U} / \mathrm{ml}$ solution of streptokinase was added to the test tubes containing $0.1 \mathrm{ml}$ of plasma and $0.65 \mathrm{ml}$ of phosphate buffer, resulting in a final concentration of $150 \mathrm{U}$ SK per $\mathrm{ml}$ of plasma. The mixture was clotted by the addition of $0.1 \mathrm{ml}$ of the stock solution of thrombin. The end point of lysis was determined visually or by a nephelometric method. The lysis time was defined as for plasma streptokinase time above. 6 Nephelometric method for recording lysis time: A Turner 111 Fluorimeter with a recorder was used to register the clotting and lysis of plasma by the SALT method. A $400 \mathrm{~nm}$ excitation filter with a $1 \%$ neutral density filter was used without an emission filter. The different light scattering properties of the plasma solution and the clot permitted the plotting of coagulation and lysis: the fluorimeter was calibrated with the plasma-SK mixture, and the return of the curve to the base line was the end point. There was close agreement between the visual and nephelometric SALT. Typical curves can be seen in Figure 5.

\section{Results}

\section{LYSIS TIME WITH DIFFERENT}

\section{CONCENTRATIONS OF SK}

Figure 1 shows the lysis time of 18 random plasma samples from inpatients as a function of increasing concentration of SK. The optimal concentration, resulting in the shortest lysis time, was found to be $500 \mathrm{U} / \mathrm{ml}$, both lower and higher concentrations prolonging the lysis time. This is in full agreement with the findings of Konttinen (1965). While there was a certain amount of scattering of lysis time with all concentrations of SK, we found that 150 $\mathrm{U} / \mathrm{ml}$ of plasma gives an optimal range of results in normal individuals and a sufficient scattering of lysis time in patients, and adopted this concentration for the SALT.

\section{INFLUENCE OF FIBRINOGEN AND \\ PLASMINOGEN CONCENTRATIONS ON \\ LYSIS TIMES}

Human fibrinogen was added to plasma, resulting in concentrations of $3 \cdot 7-16 \cdot 5 \mathrm{~g} / 1$. To each test tube containing $0.1 \mathrm{ml}$ of fibrinogen-enriched plasma $0 \cdot 2-0 \cdot 6 \mathrm{U}$ of plasminogen was added and the test was performed as for SALT. Figure 2 shows that the lysis time is getting longer as the fibrinogen concentration rises, and is shortened by increasing the plasminogen contents.

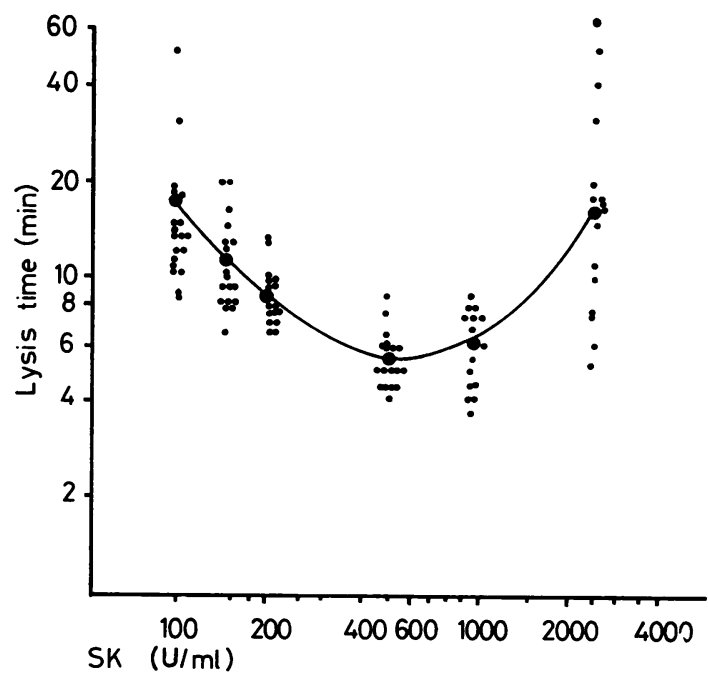

Fig. 1 Plasma lysis time as a function of SK concentrations. Average of 18 determinations plotted on logarithmic paper.

\section{STREPTOKINASE ACTIVATED LYSIS TIME}

(SALT).

Figure 3 shows the results of the SALT test in 60 normal individuals. The mean lysis time and standard deviation is $6.9 \pm 1.4 \mathrm{~min}$.

Figure 4 shows pathologically delayed fibrinolysis after myocardial infarction as determined by SALT. The details of this study have been published separately (Gidron et al., 1977); here it is intended to show the high sensitivity of this method in detecting even minor delays in fibrinolysis.

Figure 5 compares the lysis times of plasma activated by low and high SK concentrations and by urokinase on several days after myocardial infarction in a typical patient. It can be seen that the lysis time is prolonged on day 5 no matter what activation is used. There is a parallel delay in lysis in the dilute blood clot method.

\section{Discussion}

The proposed method appears to reflect the result of the interplay of all components of the fibrinolytic system with the exception of the activators, which are added in excess. It thus seems to be a sensitive screening test for deficient fibrinolytic activity in vivo. For screening purposes it is immaterial whether fibrinolysis is delayed because of a high level of fibrinogen, deficient plasminogen or a rise in inhibitors. However, a sensitive test for determining the level of natural activators is 


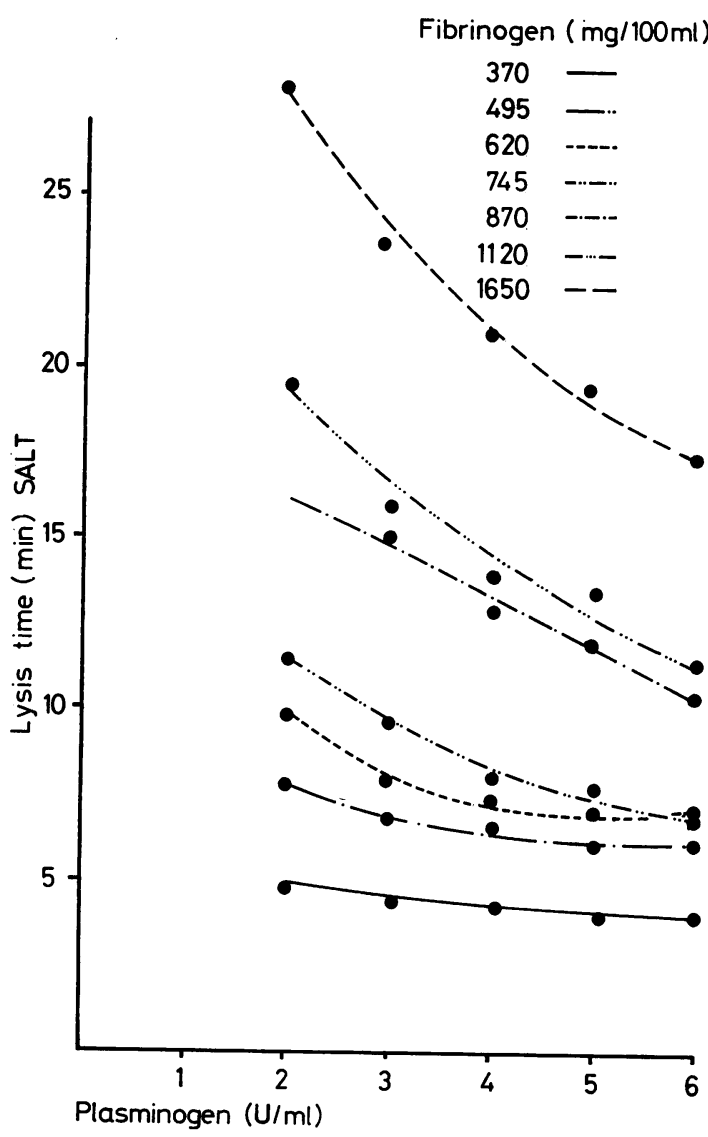

Fig. 2 Influence of plasma plasminogen and fibrinogen levels on SALT.

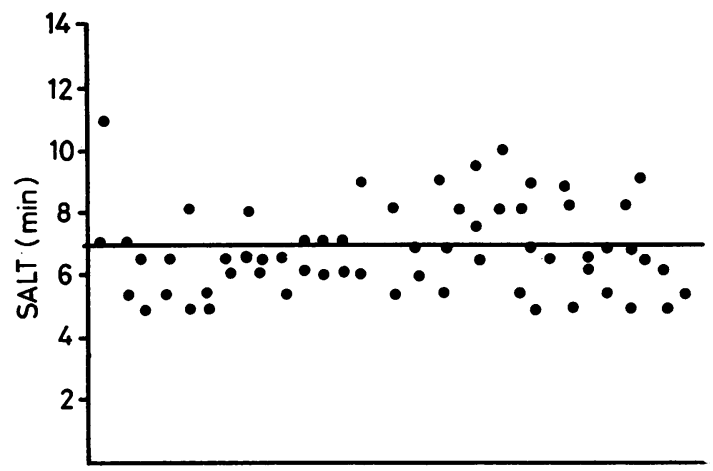

Fig. 3 Streptokinase activated lysis time (SALT) in 60 normal individuals.

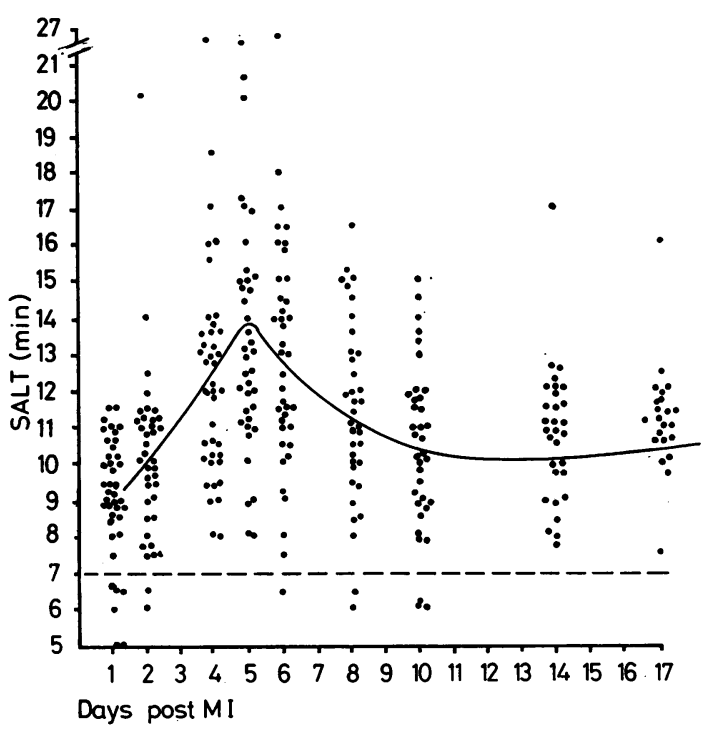

Fig. 4 Streptokinase activated lysis time in 40 patients during the postinfarction period. Broken line represents the average normal SALT.

required to complete the picture; we recently described a suitable method (Margalit et al., 1977).

The interrelations between fibrinogen and plasminogen levels and their influence on lysis time are shown in Fig. 2; the rise in plasminogen level observed by us (Gidron et al., 1977) after myocardial infarction may be part of a homoeostatic mechanism counteracting the rise in fibrinogen and inhibitor levels which precedes it. Similarly, the scattering of the lysis time in normals and random patients, seen in the SALT test, may be due to variations in the levels of the various components of the fibrinolytic system; it is unlikely that it is due to varying antistreptokinase levels because the use of higher concentrations of SK $(2500 \mathrm{U} / \mathrm{ml})$ resulted in similar scattering. Moreover, $150 \mathrm{U} / \mathrm{ml}$ of SK correspond to a dose of approximately $500000 \mathrm{U}$ in an adult, more than sufficient to neutralise any anti-SK levels likely to occur, except those after streptokinase treatment. We considered that the high concentrations of SK introduce an additional extraneous factor into the reaction and preferred the activation of the system by $150 \mathrm{U}$ SK per ml of plasma. In cases showing marked delay in lysis a control test with $2500 \mathrm{U}$ $\mathrm{SK} / \mathrm{ml}$ may be performed if there is reason to suspect a high anti-SK titre. The mean lysis time and standard deviation with this concentration is $6.7 \pm 1.9 \mathrm{~min}$. 

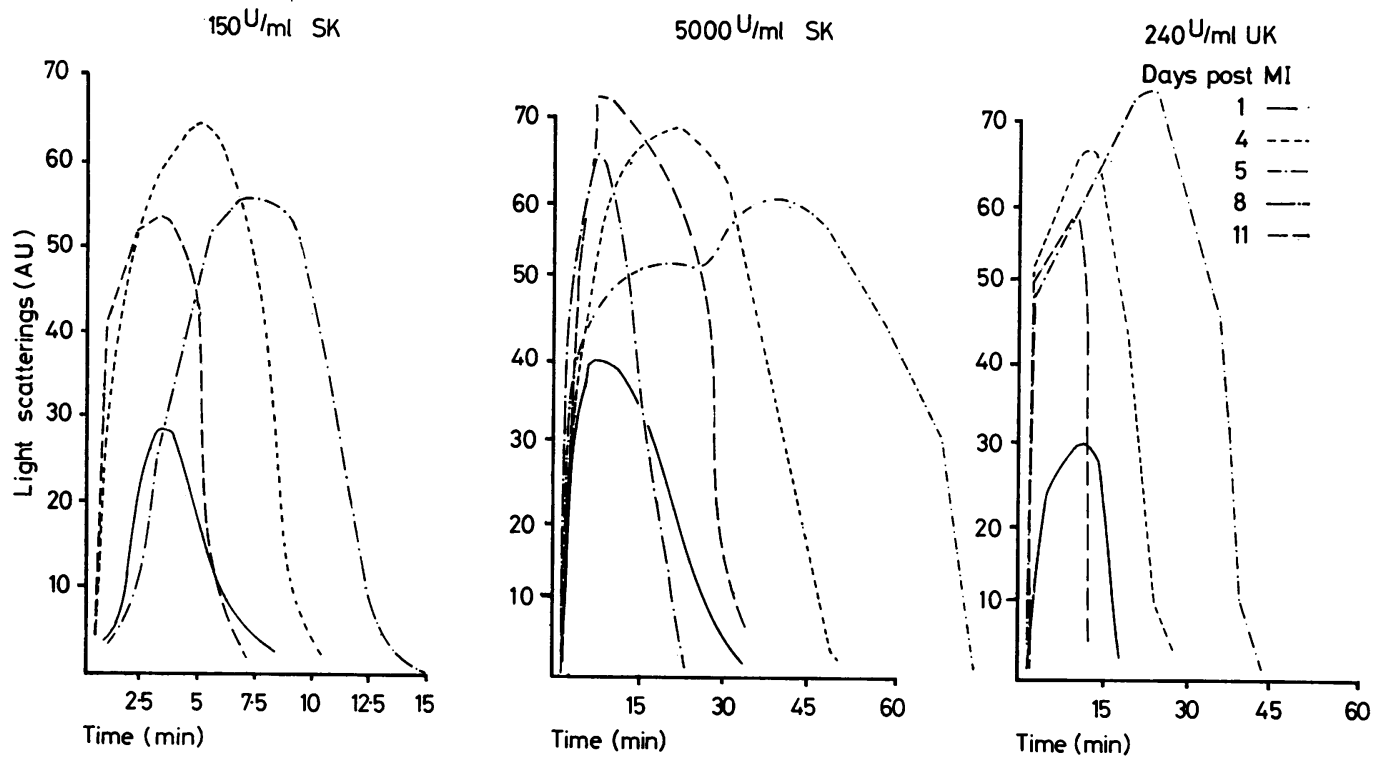

Fig. 5 Plasma lysis time on days 1, 4, 5, 8, and 11 after myocardial infarction (nephelometric recording).

\section{References}

Astrup, T., and Müllertz, S. (1952). The fibrin plate method for estimating fibrinolytic activity. Archives of Biochemistry and Biophysics, 40, 346-351.

Fearnley, G. R., Balmforth, G., and Fearnley, E. (1957). Evidence of a diurnal fibrinolytic rhythm: with a simple method of measuring natural fibrinolysis. Clinical Science, 16, 645-650.

Gidron, E., Margalit, R., Oliven, A., and Shalitin, Y. (1977). Effect of myocardial infarction on components of fibrinolytic system. British Heart Journal, 39, 19-24.

Godal, H. C. (1961). Simple syneresis procedure for fibrinogen assay (Letter). Scandinavian Journal of Clinical and Laboratory Investigation, 13, 530.

Hickman, J. A. (1971): A new technique for the quantitative estimation of fibrinolysis using in vivo ${ }^{\left[{ }^{25} \mathrm{I}\right]}$ fibrinogen. British Journal of Haematology, 20, 611-621.

von Kaulla, K. N., and Schultz, R. L. (1958): Methods for the evaluation of human fibrinolysis: studies with two combined technics. American Journal of Clinical Pathology, 29, 104-112.

Konttinen, Y. P. (1965): Activation of the fibrinolytic system in human blood by streptokinase and changes in this system and in the euglobulin clot lysis time in relation to age and sex. Thesis, University of Helsinki. Scandinavian Journal of Clinical and Laboratory Investigation, 17, Supplement, 82.

McNicol, G. P., Gale, S. B., and Douglas, A. S. (1963). In-vitro and in-vivo studies of a preparation of urokinase. British Medical Journal, 6, 909-915.

Margalit, R., Gidron, E., and Shalitin, Y. (1977): A new method for the determination of plasma activators of fibrinolysis. Thrombosis and Haemostasis, 37, 210-215. 